\title{
Crescimento de tambaqui em resposta a densidade de estocagem em tanques-rede
}

\author{
Carlos Alberto da SILVA ${ }^{1 *}$, Rodrigo Yudi FUJIMOTO ${ }^{1}$ \\ 1 Empresa Brasileira de Pesquisa Agropecuária. Av. Beira Mar, n. 3.250, Bairro Jardins, 49.025-040, Aracaju, SE, Brasil \\ * Autor correspondente: carlos-alberto.silva@embrapa.br
}

\section{RESUMO}

O tambaqui Colossoma macropomum é criado em diferentes sistemas e ambientes com boa produção em tanques-rede. O objetivo deste trabalho foi avaliar o crescimento do tambaqui em diferentes densidades de estocagem em tanques-rede em lago abastecido com água de chuva e seus efeitos sobre a qualidade ambiental. O experimento foi conduzido em fases consecutivas: recria nas densidades de 50, 100, 200 e 300 alevinos $\mathrm{m}^{-3}$ e terminação nas densidades de 20, 40, e 60 juvenis $\mathrm{m}^{-3}$. A temperatura da água, oxigênio dissolvido, $\mathrm{pH}$ e condutividade da água foram medidas diariamente. Amostras de sedimento e água foram coletadas para análise de nitrogênio e fósforo mensalmente. $\mathrm{Na}$ recria, a produção de $14,03 \mathrm{~kg} \mathrm{~m}^{-3}$ foi significativamente maior na densidade de 300 alevinos $\mathrm{m}^{-3}$. O maior peso final foi obtido na densidade de 50 alevinos $\mathrm{m}^{-3}$. A sobrevivência na recria foi superior a $97 \%$. Na terminação, o ganho de peso e o crescimento específico foram inversamente proporcionais as densidades. A conversão alimentar e o fator de condição não foram influenciados pelas densidades testadas. A densidade de 20 juvenis $\mathrm{m}^{-3}$ é a mais adequada para a terminação de tambaquis em tanques-rede. A criação de tambaqui em tanques-rede não alterou os níveis de nitrogênio e fósforo da água do lago. Aumento dos teores de matéria orgânica, nitrogênio e fósforo foram verificados no sedimento abaixo dos tanques-rede, sem causar eventos de eutrofizaçáo. O presente estudo demonstrou que a criação de tambaquis em tanques-rede é viável em lagos abastecidos com água de chuva e pode integrar-se aos preceitos dos usos múltiplos da água.

PALAVRAS-CHAVE: produção, Colossoma macropomum, nitrogênio, fósforo, qualidade ambiental

\section{Tambaqui growth in response to stocking density in cages}

\section{ABSTRACT}

The tambaqui Colossoma macropomum is rearing on different systems and environments and has shown good production in cages. The aim of this study was to evaluate the growth of tambaqui at different stocking densities in a lake supplied by rainwater and its effects on environmental quality. The experiment was conducted in consecutive phases: nursery at densities of 50, 100, 200 and 300 fingerlings $\mathrm{m}^{-3}$ and growth at densities of 20, 40 and 60 juveniles $\mathrm{m}^{-3}$. Water temperature, dissolved oxygen, $\mathrm{pH}$ and conductivity were monitored daily. Sediment and water samples were collected for analyses of nitrogen and phosphorus monthly. In the nursery, yield of $14,03 \mathrm{~kg} \mathrm{~m}^{-3}$ was significantly higher for fish at 300 fingerlings $\mathrm{m}^{-3}$. The highest weight gain was obtained at 50 fingerlings $\mathrm{m}^{-3}$. Survival over $97 \%$ was observed in the nursery. In the growth phase, weight gain and specific growth were inversely proportional to densities. Feed conversion and condition factor were not influenced by the evaluated densities. The density of 20 juveniles $\mathrm{m}^{-3}$ is more adequate to grow tambaqui in cages. Tambaqui cage culture did not alter the levels of nitrogen and phosphorus nutrients of lake water. Increased levels of organic matter, nitrogen and phosphorus in the sediment below the cages were verified without creating events of eutrophication. The results show that the tambaqui cage culture is viable in lakes provided with rainwater and can be integrated with multiple-use water services.

KEYWORDS: production, Colossoma macropomum, nitrogen, phosphorus, environmental quality 


\section{INTRODUÇÃO}

O tambaqui Colossoma macropomum (Cuvier 1818) é uma espécie nativa da Bacia Amazônica com expressiva produção em cativeiro nas regiōes Norte e Nordeste, que é criada em diferentes sistemas e ambientes: tanques-redes em várzeas inundáveis, remanso de rios e barragens; viveiros escavados e canais de irrigação (Gomes et al. 2010; Silva et al. 2013). A criação de tambaquis em tanques-rede pode ser uma alternativa viável para a produção de pescado em pequenos lagos e açudes comunitários destinados a captação de água pluvial para consumo doméstico, irrigação e dessedentação animal; integrando aos usos múltiplos da água existentes nesses reservatórios sem a necessidade de drenagem para a despesca. Adicionalmente, é importante conhecer o impacto do sistema de produçáo de peixes em tanques-rede sobre a qualidade do ambiente aquático para atender o multiuso e garantir a sustentabilidade.

A criação de peixes é fonte de nutrientes inorgânicos e orgânicos para o ambiente aquático na forma de restos de ração nấo consumida, excretas e fezes dos peixes (Pillay 2004). Um dos mais citados impactos é o aumento da carga orgânica nos sedimentos abaixo dos tanques-rede e o aumento do nitrogênio e fósforo total nos sedimentos e água intersticial que podem causar eutrofização e interferir nas comunidades bentônicas (Karakassis et al. 2000; Molina et al. 2001; La Rosa et al. 2004). A magnitude do impacto no ambiente aquático depende basicamente da intensificação do sistema de produção dos peixes, dispersão do material particulado e dissolvido pelas correntes e da capacidade de suporte para assimilar as cargas orgânicas de nitrogênio e fósforo, principalmente (AguadoGiménez e García-Garcia 2004).

Por outro lado, os benefícios da criaçáo em tanques-rede são redução do espaço físico necessário à criação, maior controle e aproveitamento eficiente de raçáo, diminuição dos custos de produção, maior produtividade sem aumento da área, menor investimento inicial $(70 \%$ menor que viveiros convencionais) e maior facilidade na despesca (Conte et al. 2008), tornando a atividade mais competitiva e sustentável. Assim, para maximizar a produção e a lucratividade, densidades de estocagem ideais necessitam ser determinadas para cada espécie de peixe e fase de desenvolvimento. A densidade de estocagem é considerada um fator crítico na produção final devido à influência na sobrevivência, crescimento dos peixes, produção e qualidade da água (Oliveira et al. 2007; Rowland et al. 2006). Os resultados do desempenho zootécnico de tambaquis em sistemas de produção aquícola controlados aliados aos seus efeitos na qualidade ambiental são inexistentes na literatura e pouco difundidos ao setor produtivo. Deste modo, o objetivo deste trabalho foi avaliar o crescimento do tambaqui em diferentes densidades de estocagem nas fases de recria e terminaçáo e seu efeito na qualidade ambiental visando subsidiar a produção de peixes com peso comercial de 1,0 kg.

\section{MATERIAL E MÉTODOS}

O experimento foi conduzido no lago escavado em terreno natural de 0,55 ha localizado na Embrapa Tabuleiros Costeiros em Aracaju/SE. O lago não possuía nenhum uso, ictiofauna e vegetação aquática até o início do experimento. $\mathrm{O}$ lago foi abastecido com água da chuva, sendo que a profundidade no local de fixação dos tanques-rede variou entre 1,4 a 2,5 m de acordo com a época do ano. $\mathrm{O}$ experimento teve a duraçáo de 98 dias para a recria e de 270 dias para a terminação, e foi conduzido entre outubro de 2011 e outubro de $2012 \mathrm{em}$ tanques-rede flutuantes de $1 \mathrm{~m}^{3}(1,0 \times 1,0 \times 1,0 \mathrm{~m})$ espaçados de $1,0 \mathrm{~m}$ e construídos com tela metálica com abertura de malha de $20 \mathrm{~mm}$ e cobertos com telas anti-pássaros. $\mathrm{Na}$ recria foram utilizados bolsōes de nylon de malha $4 \mathrm{~mm}$ no interior dos tanques-rede que serviram como berçários para a retençáo dos alevinos de peso médio inicial de $0,35 \pm 0,02$ g obtidos na Estaçáo de Piscicultura do Itiubá -CODEVASF (Companhia de Desenvolvimento dos Vales do São Francisco e do Parnaíba), localizada no município de Porto Real do Colégio - AL. O delineamento experimental foi inteiramente casualizado (DIC), constituído de quatro tratamentos 50, 100,200 e 300 alevinos $\mathrm{m}^{-3}$ e três repetiçóes. Os alevinos na recria foram alimentados seis dias na semana e quatro vezes por dia $(7: 00 ; 10: 00 ; 13: 00$ e 16:00) com ração extrusada comercial para peixes onívoros com teores de proteína bruta de 40 e $36 \%$ em diferentes granulometrias (farelada; 1,7 e 3 $\mathrm{mm}$ ). A medida que os peixes cresciam foi fornecida ração de menor teor protéico e de maior granulometria. A taxa de alimentaçáo variou de 15 a $6 \%$ do peso vivo dos peixes com base nas indicaçôes de fornecimento/uso da ração descritas na embalagem do fabricante.

Imediatamente após a finalização da recria iniciou-se o experimento de terminaçáo com os juvenis de tambaqui com peso médio de 73,8 $\pm 2,5 \mathrm{~g}$ aleatoriamente coletados e provenientes do experimento prévio de recria no mesmo lago. O delineamento experimental utilizado foi inteiramente casualizado (DIC) nas densidades de estocagem 20, 40 e 60 juvenis $\mathrm{m}^{-3}$ com quatro repetiçóes. Na terminação, os peixes foram alimentados seis dias na semana e duas vezes ao dia (9:00 e 16:00) com ração extrusada comercial para peixes onívoros com teores de proteína bruta de 36,32 e $28 \%$ e diferentes granulometrias $(3,4,6$ e $10 \mathrm{~mm})$. A medida que os peixes cresciam foi fornecida raçấo de menor teor protéico e de maior granulometria. A taxa de alimentação variou de 3 a $1 \%$ do peso vivo dos peixes com base nas indicaçóes de fornecimento/uso da ração descritas na embalagem do fabricante. As biometrias foram realizadas a cada 30 dias com uma amostra de $10 \%$ da populaçáo em cada tanque-rede e 
serviram para ajustar a quantidade e a granulometria da ração a ser fornecida mensalmente e avaliar o crescimento em peso.

Ao término do experimento, os parâmetros de avaliação do desempenho zootécnico avaliados foram: ganho em peso total (g) (GPT): diferença entre a média de peso dos peixes no final e no início do período experimental; ganho em peso/dia $\left(\mathrm{g} \mathrm{dia}^{-1}\right)$ (GPD): diferença entre a média de peso dos peixes no final e no início do período experimental dividido pelo número de dias do período experimental; taxa de crescimento específico (\% peso dia $\left.^{-1}\right)$ (TCE): relação percentual entre a diferença do logaritmo neperiano da média de peso dos peixes no final e no início do período experimental dividido pelo número de dias do experimento; conversão alimentar aparente (CAA): relação entre a média de consumo de ração e a média de ganho em peso durante o período experimental; taxa de sobrevivência (\%) (TS): relação percentual entre o número de peixes no final e no início de cada etapa experimental e fator de condição $(\mathrm{K})$ : relaçáo entre o peso e o comprimento padrão dos peixes em um determinado tempo.

Os parâmetros de qualidade de água como temperatura, $\mathrm{pH}$, oxigênio dissolvido e condutividade foram monitorados in situ no ponto central da linha de tanques-rede seis vezes por semana pela manhã e tarde nos horários da alimentação utilizando-se uma sonda multiparamétrica (Horiba U-50, Kyoto, Japan) sendo a medição neste local representativa para todas as unidades experimentais. Para avaliar o impacto ambiental do sistema de produção em tanques-rede, água e sedimento de fundo do lago foram coletados mensalmente em dois pontos: um situado no centro da linha dos tanques-rede e outro (controle) afastado $50 \mathrm{~m}$ em direção perpendicular a linha de tanques. A água do lago foi coletada com garrafa de Van Dorn a $20 \mathrm{~cm}$ abaixo da linha de água. Em seguida, foi acondicionada em garrafas de polietileno de $500 \mathrm{~mL}$ previamente limpas e descontaminadas com solução $10 \%$ v/v de $\mathrm{HCl}$. Foram determinadas as concentraçóes na água de nitrogênio e fósforo total de acordo com APHA (2005) e dureza total da água por espectrofotometria de absorção atômica (SpectrAA 55B, Varian, Melbourne, Australia) de acordo com Primavesi et al. 2005a. O sedimento foi coletado com amostrador tipo "corer" construído em tubo de pvc 40 $\mathrm{mm}$ de diâmetro e $50 \mathrm{~cm}$ de comprimento. Após a coleta, o sedimento sofreu secagem ao ar, tamização e homogeneização. $\mathrm{Na}$ análise do fósforo no sedimento foi utilizada a solução duplo-ácida extratora de Mehlich, e sua concentração determinada por espectrofotometria a $660 \mathrm{~nm}$ (Primavesi et al. 2005b). $\mathrm{Na}$ análise do nitrogênio total no sedimento, a amostra foi submetida ao método Kjeldahl e sua concentração medida por titulação com $\mathrm{H}_{2} \mathrm{SO}_{4}$ (Primavesi et al. 2005b).

Os resultados foram expressos como média \pm desvio padrão. Inicialmente, verificou-se a normalidade dos dados pelo teste de Shapiro-Wilk. Em seguida, verificou-se a homogeneidade das variâncias utilizando o teste de Barlett. Os dados foram submetidos à análise de variância e quando verificadas diferenças significativas $(p<0,05)$ foi aplicado o teste de Tukey para comparação das médias. Os dados foram processados pelo software Sisvar 5.1 Build 72 (Ferreira 2011). A regressão linear foi empregada para avaliar os efeitos da densidade de estocagem sobre os parâmetros do desempenho zootécnico obtidos nesse estudo.

\section{RESULTADOS}

A temperatura da água variou de 25,5 a $32,2{ }^{\circ} \mathrm{C}$ com a média de $29,1 \pm 1,4^{\circ} \mathrm{C}$. A máxima foi registrada no mês de dezembro à tarde, com diminuição gradual atingindo o valor mínimo em agosto pela manhã. Foi registrado um pequeno aumento da temperatura à tarde com acréscimo médio de 0,7 a $1,2^{\circ} \mathrm{C}$ (Figura $1 \mathrm{~A}$ ). $\mathrm{O} \mathrm{pH}$ variou entre 4,0 a $8,8 \mathrm{com}$ média $6,5 \pm 1,1$. O valor máximo foi atingido após 120 dias da calagem inicial com $2.500 \mathrm{~kg}$ de calcário agrícola aplicado no lago no início da fase berçário. Não foi observada diferença entre os valores do $\mathrm{pH}$ de manhá e a tarde (Figura 1B). O oxigênio dissolvido (OD) variou entre 6,1 a $10,3 \mathrm{mg} \mathrm{L}^{-1}$ com média $8,3 \pm 0,8 \mathrm{mg} \mathrm{L}^{-1}$. Elevação de no máximo $1,2 \mathrm{mg} \mathrm{L}^{-1}$ no OD foi registrada no período da tarde ao longo da criação (Figura 1C). A condutividade da água variou entre 442,2 a $699,9 \mu \mathrm{S} \mathrm{cm}^{-1}$ com média de $606,2 \pm 69,9 \mu \mathrm{S} \mathrm{cm}^{-1}$. O aumento da condutividade foi registrado ao longo dos seis primeiros meses da estação seca de verão e alcançou o valor máximo no mês seguinte ao início do período chuvoso. Não foi observada diferença entre os valores da condutividade de manhã e a tarde (Figura 1D). O nitrogênio total na água (NTA) variou de 0 a $0,04 \mathrm{mg} \mathrm{L}^{-1}$ no ponto central dos tanques-rede e de 0 a 0,03 $\mathrm{mg} \mathrm{L}^{-1}$ na estação controle, e não apresentaram diferenças. Os valores do NTA apresentaram um pequeno aumento no ponto central da linha dos tanques-rede no mês anterior ao início do período chuvoso. O fósforo total na água (FTA) variou de 0,2 a $0,8 \mathrm{mg} \mathrm{L}^{-1}$ e média de $0,3 \pm 0,2 \mathrm{mg} \mathrm{L}^{-1}$ no ponto central dos tanques-rede e de 0 a $0,8 \mathrm{mg} \mathrm{L}^{-1}$ e média de $0,3 \pm 0,2 \mathrm{mg} \mathrm{L}^{-1}$ na estação controle. Os valores do FTA também não apresentaram diferenças entre os dois pontos de coleta. A dureza total da água variou de 216,4 a 555,0 $\mathrm{mg} \mathrm{L}^{-1}$ com média de 364,8 \pm 122,0 $\mathrm{mg} \mathrm{L}^{-1}$. O valor máximo ocorreu quatro meses após a aplicação da calagem sendo seguido de uma queda gradual com o início do período chuvoso na região. A matéria orgânica (MO) no sedimento variou de 9,3 a 85,0 $\mathrm{g} \mathrm{kg}^{-1}$ com média de 41,1 \pm $27,5 \mathrm{~g} \mathrm{~kg}^{-1}$ no ponto central dos tanques-rede e de 4,1 a 46,8 $\mathrm{g} \mathrm{kg}^{-1}$ e média de $19,1 \pm 13,7 \mathrm{~g} \mathrm{~kg}^{-1}$ na estação controle. Os dois pontos apresentaram diferenças significativas em relação ao teor de $\mathrm{MO}(\mathrm{p}<0,05)$. O nitrogênio total do sedimento (NTS) abaixo dos tanques-rede no ponto central variou de 0,36 a 1,61 $\mathrm{g} \mathrm{kg}^{-1}$ com média de 0,96 $\pm 0,41 \mathrm{~g} \mathrm{~kg}^{-1}$. O nitrogênio total do sedimento no ponto controle variou de 0,18 a $1,05 \mathrm{~g}$ 

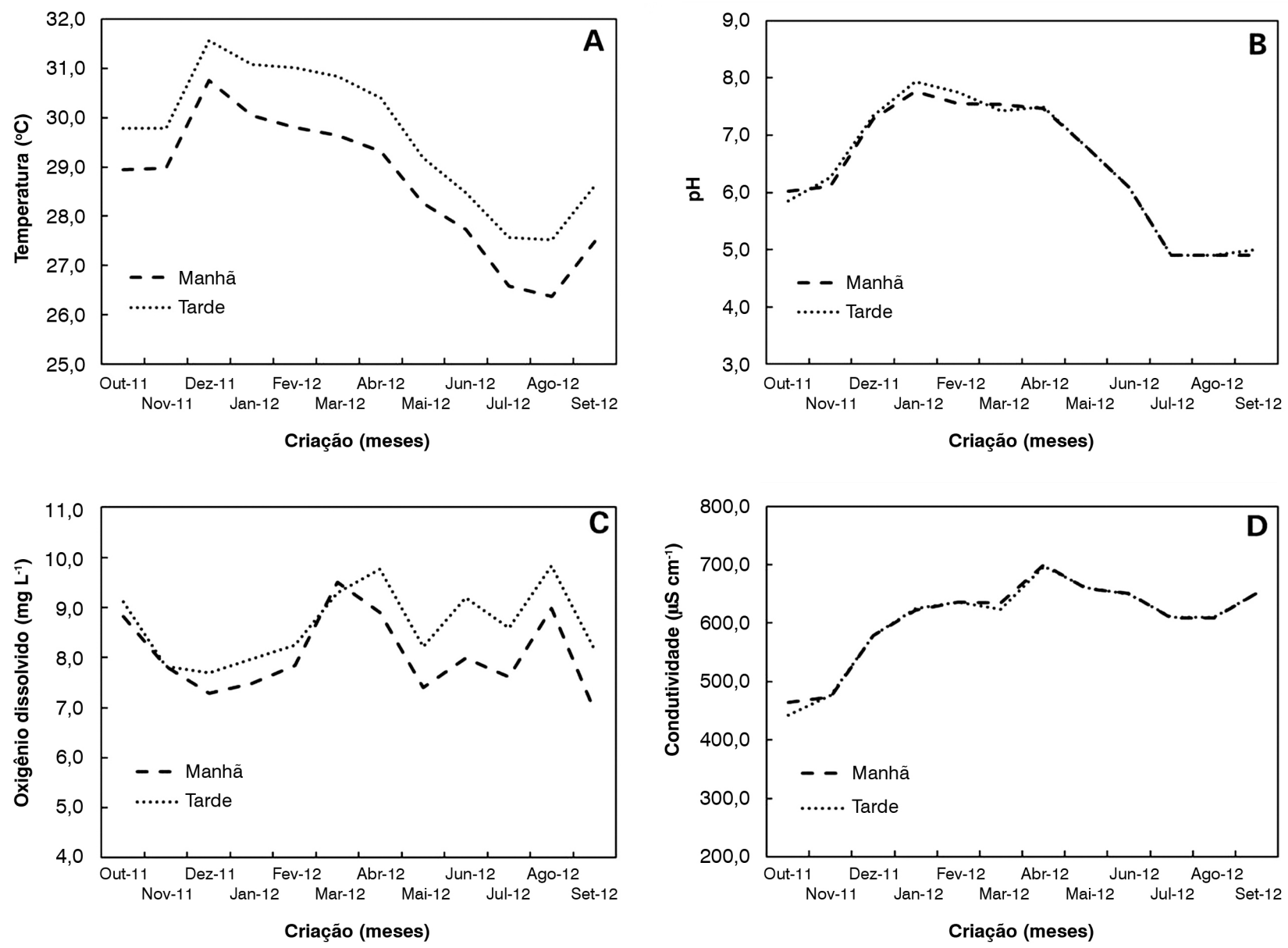

Figura 1. Variação mensal da temperatura (A), pH (B), oxigênio dissolvido (C) e condutividade elétrica (D) da água do lago.

$\mathrm{kg}^{-1}$ com média de $0,41 \pm 0,26 \mathrm{~g} \mathrm{~kg}^{-1}$. Os valores do NTS abaixo dos tanques-rede foram maiores em todas as coletas (Figura 2) e diferem significativamente do ponto controle $(\mathrm{p}<0,05)$. O fósforo total do sedimento (FTS) abaixo dos tanques-rede no ponto central variou de 5,37 a 19,10 $\mathrm{mg} \mathrm{kg}^{-1}$ com média de 10,7 $\pm 4,9 \mathrm{mg} \mathrm{kg}^{-1}$. O fósforo total do sedimento no ponto controle variou de 3,62 a 11,98 $\mathrm{mg} \mathrm{kg}^{-1}$ com média de 6,1 $\pm 2,6 \mathrm{mg}$ $\mathrm{kg}^{-1}$. Os valores do NTS abaixo dos tanques-rede foram maiores que no ponto controle com exceçáo dos meses de Novembro e Dezembro de 2011 e Março de 2012 (Figura 3).

A conversão alimentar aparente (CAA) foi significativamente menor nas densidades de 300 e 100 alevinos $\mathrm{m}^{-3}$ em comparação com 50 e 200 alevinos $\mathrm{m}^{-3}$ (Tabela 1). A melhor conversão alimentar de 0,8 foi obtida na maior densidade 300 alevinos $\mathrm{m}^{-3} \mathrm{e}$ todos os tratamentos apresentaram valores próximos à unidade variando de 0,8 a 1,1 (Tabela 1). A regressão linear da CAA com a densidade de estocagem na recria apresentou baixo coeficiente de determinação $\mathrm{R}^{2}$ de 0,57 . O peso final

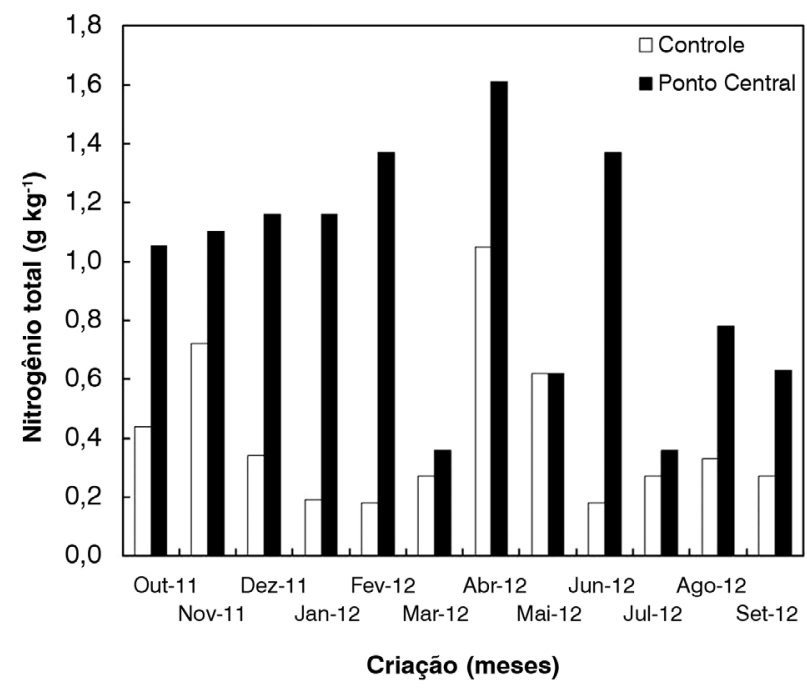

Figura 2. Variação mensal do nitrogênio total do sedimento abaixo dos tanques-rede. 


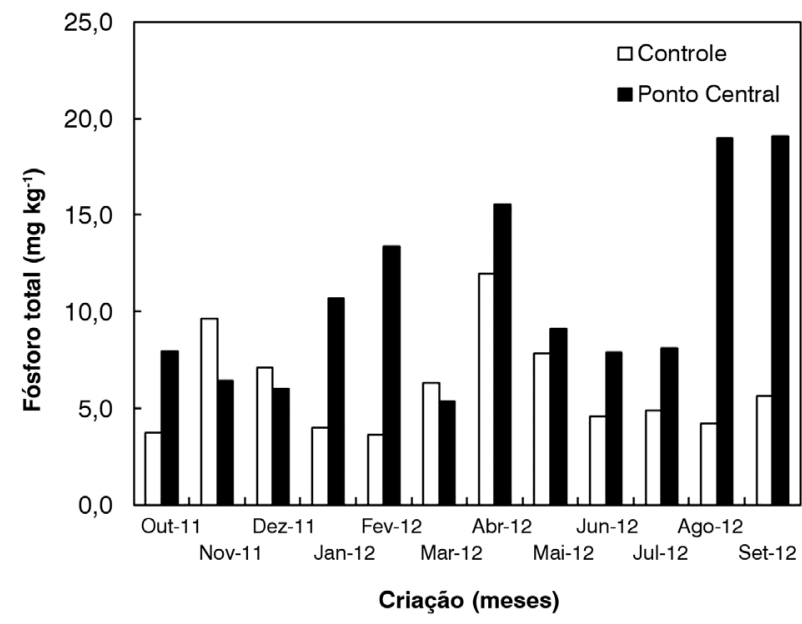

Figura 3. Variação mensal do fósforo total do sedimento abaixo dos tanques-rede.

Tabela 1. Valores (média \pm desvio-padrão) dos parâmetros zootécnicos avaliados nas diferentes densidades de estocagem na fase de recria.

\begin{tabular}{lcccc}
\hline \multirow{2}{*}{ Parâmetro } & \multicolumn{4}{c}{${\text { Alevinos } \mathrm{m}^{-3}}$} \\
\cline { 2 - 5 } & 50 & 100 & 200 & 300 \\
\hline $\begin{array}{l}\text { Conversão } \\
\text { alimentar }\end{array}$ & $1,1 \pm 0,1^{\mathrm{b}}$ & $0,9 \pm 0,1^{\mathrm{a}}$ & $1,0 \pm 0,1^{\mathrm{b}}$ & $0,8 \pm 0,1^{\mathrm{a}}$ \\
$\begin{array}{l}\text { Peso final (g) } \\
83,5 \pm 18,0^{\mathrm{a}}\end{array}$ & $70,8 \pm 19,1^{\mathrm{a}}$ & $44,0 \pm 4,7^{\mathrm{b}}$ & $46,8 \pm 9,2^{\mathrm{b}}$ \\
$\begin{array}{l}\text { Sobrevivência } \\
\text { (\%) }\end{array}$ & $88,2 \pm 13,5^{\mathrm{b}}$ & $97,4 \pm 2,2^{\mathrm{a}}$ & $99,5 \pm 0,6^{\mathrm{a}}$ & $99,7 \pm 0,4^{\mathrm{a}}$ \\
$\begin{array}{l}\text { Produção } \\
\left.\text { (kg m }{ }^{-3}\right)\end{array}$ & $3,57 \pm 1,91^{\mathrm{c}}$ & $6,89 \pm 1,5^{\mathrm{b}}$ & $8,79 \pm 0,93^{\mathrm{b}}$ & $14,03 \pm 2,75^{\mathrm{a}}$ \\
$\begin{array}{l}\text { a-c médias seguidas por letras minúsculas diferentes nas linhas, diferem } \\
\text { estatisticamente entre si }(\mathrm{P}<0,05) .\end{array}$
\end{tabular}

dos alevinos nas duas menores densidades 50 e 100 alevinos $\mathrm{m}^{-3}$ não apresentou diferenças significativas da mesma maneira do que foi observado nas duas maiores densidades 200 e 300 alevinos $\mathrm{m}^{-3}$ (Tabela 1). Entretanto, as menores densidades de estocagem $50 \mathrm{e} 100$ alevinos $\mathrm{m}^{-3}$ proporcionaram um peso final significativamente maior que nas maiores densidades $(\mathrm{p}<0,05)$. $\mathrm{O}$ peso final apresentou relaçáo inversa com a densidade de estocagem, sendo que o maior peso final de $83,2 \mathrm{~g}$ foi atingido pelos peixes criados na densidade de 50 alevinos $\mathrm{m}^{-3}$ (Figura 4A, Tabela 1). A sobrevivência média final na recria foi superior a $97 \%$ nas três maiores densidades. A sobrevivência média de $88,2 \%$ na menor densidade de 50 alevinos $\mathrm{m}^{-3}$ foi menor devido a mortalidade por estresse no povoamento em uma das repetiçóes atingindo $68 \%$ de sobrevivência. A produção na fase de recria apresentou relação direta com a densidade de estocagem (Figura 4B). A produção média final de $14,03 \mathrm{~kg} \mathrm{~m}^{-3}$ obtida na densidade de 300 alevinos $\mathrm{m}^{-3}$ foi significativamente maior quando comparada com as demais densidades avaliadas (Tabela 1). Comportamento semelhante foi observado para a terminaçáo onde o peso médio final de 1028,5 g observado na menor densidade de 20 juvenis $\mathrm{m}^{-3}$ foi significativamente maior que o da maior densidade de 60 juvenis $\mathrm{m}^{-3}(\mathrm{p}<0,05)$, mas não diferiu de $901,8 \mathrm{~g}$ obtido no tratamento de 40 juvenis $\mathrm{m}^{-3}$ (Tabela 2).

$\mathrm{O}$ ganho em peso apresentou relação inversa com a densidade de estocagem (Figura 5A), sendo que o maior valor de $955,0 \pm 84,90$ g obtido na menor densidade de 20 juvenis $\mathrm{m}^{-3}$ foi significativamente maior do que o obtido na maior densidade de 60 juvenis $\mathrm{m}^{-3}(\mathrm{p}<0,05)$, mas não diferiu do tratamento de 40 juvenis $\mathrm{m}^{-3}$ (Tabela 2). A densidade de estocagem náo influenciou a conversáo alimentar aparente (CAA) na terminação (Figura 5B, Tabela 2). A taxa de crescimento específico (TCE) apresentou relação inversa com a densidade de estocagem (Figura $5 \mathrm{C}$ ) e foi menor que $1 \% \mathrm{dia}^{-1} \mathrm{em}$ todos os tratamentos. A TCE média variou de 0,89 a 0,98 e apresentou diferença significativa entre as densidades (Tabela 2). Durante a terminação, não houve mortalidades nos tratamentos $\mathrm{e} o$ fator de condição não sofreu influência da densidade de estocagem. A produçâo final apresentou relação direta com a densidade de estocagem (Figura 5D). A produção de $48,7 \mathrm{~kg} \mathrm{~m}^{-3}$ obtida na densidade de 60 juvenis $\mathrm{m}^{-3}$ foi significativamente maior quando comparada com as demais densidades avaliadas (Tabela 2).
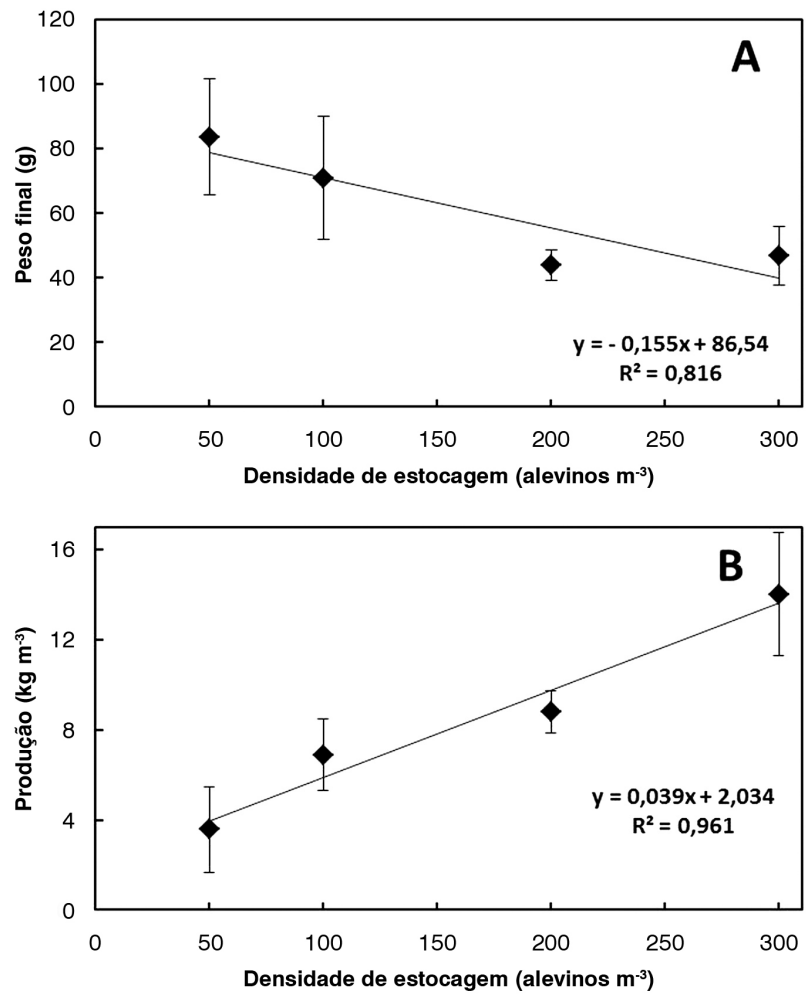

Figura 4. Relação linear entre densidade de estocagem e peso final (A) e produção (B) na fase de recria do tambaqui. 

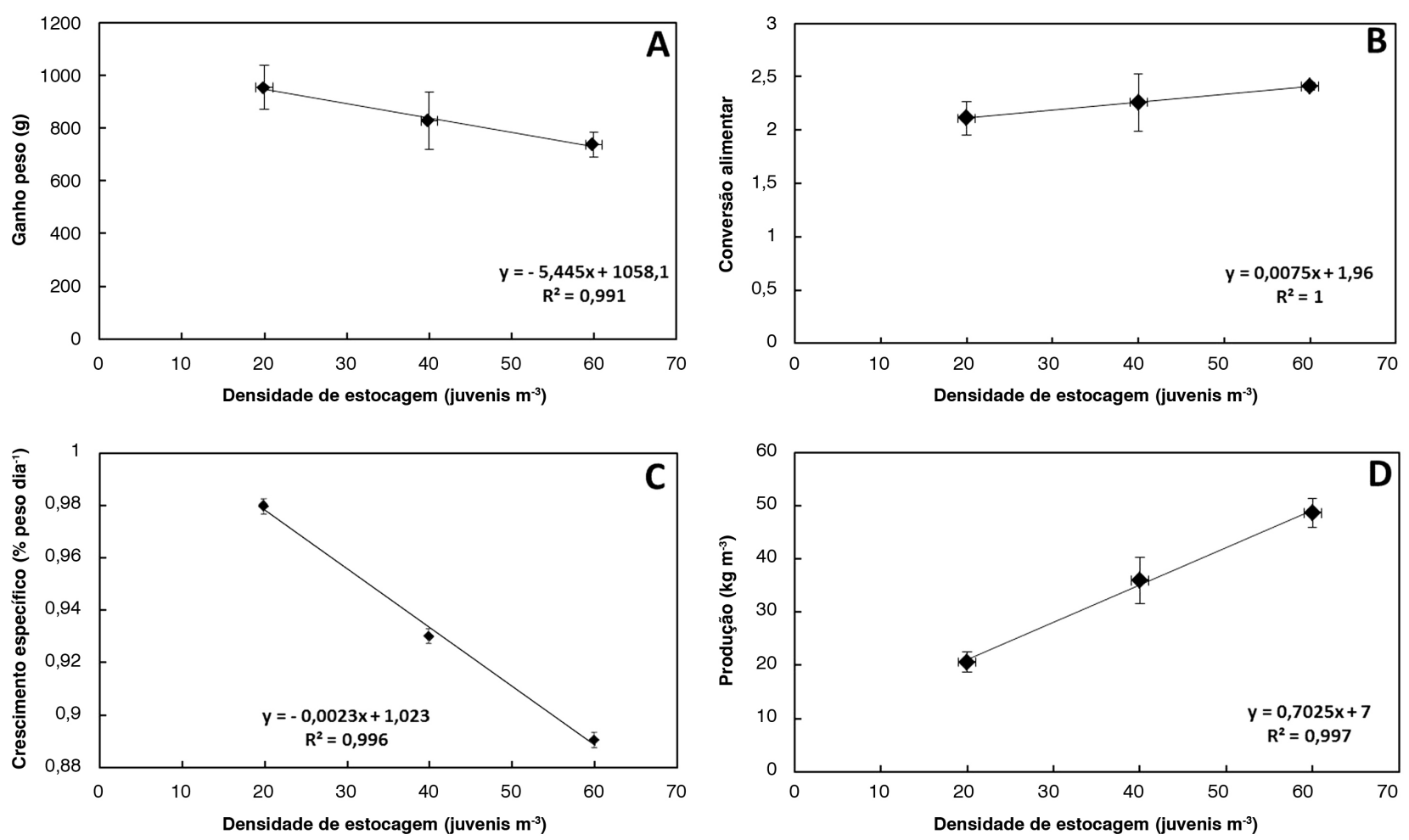

Figura 5. Relação linear entre densidade de estocagem e ganho em peso (A); conversão alimentar (B); crescimento específico (C) e produção (D) na fase de terminação do tambaqui.

Tabela 2. Valores (média \pm desvio-padrão) dos parâmetros zootécnicos avaliados nas diferentes densidades de estocagem na fase de terminação.

\begin{tabular}{|c|c|c|c|}
\hline \multirow{2}{*}{ Parâmetro } & \multicolumn{3}{|c|}{ Juvenis $\mathrm{m}^{-3}$} \\
\hline & 20 & 40 & 60 \\
\hline Peso inicial (g) & $73,5 \pm 3,26$ & $73,2 \pm 3,44$ & $74,77 \pm 0,97$ \\
\hline Peso final $(\mathrm{g})$ & $1028,5 \pm 84,90^{a}$ & $901,8 \pm 94,88^{\mathrm{ab}}$ & $812,0 \pm 39,85^{b}$ \\
\hline $\begin{array}{l}\text { Ganho em peso } \\
\text { (g) }\end{array}$ & $955,0 \pm 84,90^{a}$ & $828,6 \pm 109,55^{a b}$ & $737,2 \pm 46,02^{b}$ \\
\hline $\begin{array}{l}\text { Conversão } \\
\text { alimentar }\end{array}$ & $2,11 \pm 0,16$ & $2,26 \pm 0,27$ & $2,41 \pm 0,04$ \\
\hline $\begin{array}{l}\text { Crescimento } \\
\text { específico } \\
\left(\% \text { peso } \text { dia }^{-1}\right)\end{array}$ & $0,98 \pm 0,03^{a}$ & $0,93 \pm 0,05^{a b}$ & $0,89 \pm 0,02^{b}$ \\
\hline $\begin{array}{l}\text { Ganho em peso } \\
\text { diário }\left(\mathrm{g} \mathrm{dia}^{-1}\right)\end{array}$ & $3,5 \pm 0,36^{a}$ & $3,1 \pm 0,41^{\mathrm{ab}}$ & $2,7 \pm 0,17^{b}$ \\
\hline Sobrevivência (\%) & 100,0 & 100,0 & 100,0 \\
\hline Fator de condição & $3,8 \pm 0,19$ & $3,6 \pm 0,24$ & $3,7 \pm 0,11$ \\
\hline Produção $\left(\mathrm{kg} \mathrm{m}^{-3}\right)$ & $20,6 \pm 1,96^{a}$ & $36,0 \pm 4,38^{b}$ & $48,7 \pm 2,76^{c}$ \\
\hline
\end{tabular}

a-cmédias seguidas por letras minúsculas diferentes nas linhas, diferem estatisticamente entre si $(\mathrm{P}<0,05)$.

\section{DISCUSSÃO}

Os parâmetros ambientais da água apresentaram variaçóes dentro da faixa recomendada para a criação do tambaqui (Gomes et al. 2010; Silva 2011). O pH neste estudo apresentou grande amplitude de valores devido a calagem ter sido feita somente uma vez no início da fase berçário. A diminuição gradual do $\mathrm{pH}$ (Figura 1B) pode ser explicada pela precipitação e entrada no lago de água da chuva de $\mathrm{pH}$ ao redor de 5,5 durante o período chuvoso com precipitaçóes mais elevadas de abril a junho na regiáo de Aracaju (Costa e Souza 2010). O regime pluviométrico na região é dividido claramente em uma estação de inverno chuvosa de abril a julho e uma estação de verão seca de outubro a janeiro (SIRHSE 2012). O abastecimento do lago é exclusivamente via água de chuva que é captada na pequena bacia de contribuiçáo ao redor do lago e armazena água suficiente para transcorrer o verão seco. Durante a estaçáo seca, a profundidade no local dos tanques-rede alcançou 1,4 $\mathrm{m}$ e na estação chuvosa atingiu o máximo de 2,5 m com predominância de águas ácidas. Segundo Aride et al. (2007), o tambaqui possui relativa tolerância aos ambientes ácidos com estratégias adaptativas que envolvem ajustes hematológicos, ionrregulação e produção de muco. A resistência do tambaqui a água ácida 
reflete sua ocorrência em ambientes naturalmente ácidos como as águas da bacia amazônica (Silva et al. 2008). A elevação da condutividade nos 6 primeiros meses da criaçáo (Figura1D) pode estar relacionada ao provável aumento dos íons dissolvidos proveniente da dissolução gradual do calcário usado na calagem inicial. O valor máximo de $699,9 \mu \mathrm{S} \mathrm{cm}{ }^{-1}$ atingido após o início das chuvas pode ser explicado pela lixiviaçáo de partículas do solo ao redor do lago causado pela chuva neste período.

Os valores do nitrogênio total da água (NTA) do lago são menores que o estabelecido na resolução CONAMA n. 375/2005 para a classe 2 que se destina ao cultivo de organismos aquáticos para fins de consumo. Henry-Silva e Camargo (2006) reportaram teores médios de 0,34 \pm 0,06 $\mathrm{mg} \mathrm{L}^{-1}$ de nitrogênio total em efluentes de criaçáo de tilápias em viveiros e que são superiores ao máximo de $0,04 \mathrm{mg} \mathrm{L}^{-1}$ registrado neste estudo. Mallasen et al. (2012) observaram concentraçóes médias elevadas de NTA de $6,3 \pm 3,1 \mathrm{mg} \mathrm{L}^{-1}$ no ponto de coleta mais afastado dos 240 tanques-rede instalados no parque aquícola de Ilha Solteira, SP com entrada mensal de $141 \mathrm{t}$ de raçáo e produçáo mensal de 85 t de tilápias. Segundo os mesmos autores, além da tilapicultura, no entorno da bacia do reservatório de Ilha Solteira existem atividades agropecuárias que podem aumentar o NTA no local devido ao carreamento de nutrientes do solo e detritos orgânicos no período chuvoso.

Com relaçáo ao fósforo total da água, valores médios de $0,07 \pm 0,02 \mathrm{mg} \mathrm{L}^{-1}$ reportado por Henry-Silva e Camargo (2006) e de 0,02 $\pm 0,03 \mathrm{mg} \mathrm{L}^{-1}$ por Mallasen et al. (2012) são inferiores aos valores máximos encontrados neste estudo de $0,80 \mathrm{mg} \mathrm{L}^{-1}$. Entretanto, o valor máximo do fósforo total da água do lago de $0,80 \mathrm{mg} \mathrm{L}^{-1}$ é superior ao recomendado pela resolução CONAMA n. 375/2005 para a classe 2 de até 0,03 $\mathrm{mg} \mathrm{L}^{-1} \mathrm{em}$ ambientes lênticos, porém não foram observados estados de eutrofização da água do lago. Os maiores teores de matéria orgânica e nitrogênio observados nos sedimentos abaixo dos tanques-rede comparados ao ponto controle em todas as coletas mensais foram previstos e podem ser atribuídos a deposição de sobras de ração, excretas e metabólitos dos peixes, configurando que a ração é fonte mais importante de nutrientes para o meio (Pillay 2004; Islam 2005).

$\mathrm{O}$ aumento do fósforo total no sedimento (FTS) abaixo dos tanques-rede em relação ao controle pode ser explicado pelo teor deste nutriente na ração fornecida que contém no mínimo de 6 a $15 \mathrm{~g} \mathrm{~kg}^{-1}$ de $\mathrm{P}$ (níveis de garantia do fabricante) que deposita no fundo pelas fezes dos peixes e sobras de ração. As perdas de $\mathrm{N}$ e $\mathrm{P}$ da ração para o ambiente são expressivas podendo atingir cerca de $75 \%$ para o $\mathrm{N}$ e de 53 a $70 \%$ para o P na produção de $1 \mathrm{~kg}$ de peixe (Pillay 2004; Boyd et al. 2007). A diminuiçáo da concentraçáo de FTS abaixo dos tanques observado nos meses de menor temperatura da água pode ser atribuída à diminuição da ingestáo de alimento pelos tambaquis neste período que passaram a não se alimentar com temperaturas da água abaixo de $26^{\circ} \mathrm{C}$ e, consequentemente, menores cargas de matéria orgânica (sobras de raçáo, excretas e fezes) foram aportadas aos sedimentos. Dessa maneira, para evitar o desperdício de ração, o regime alimentar foi alterado de duas refeiçóes/dia para somente uma à tarde oferecida nos dias ensolarados em razáo da diminuiçáo do apetite dos peixes causada pela reduçáo do metabolismo (Américo et al. 2013; Silva 2011). A concentração média do FTS abaixo dos tanques-rede foi inferior aos valores médios encontrados em solos de viveiros de camaróes de $859 \pm 158 \mathrm{mg} \mathrm{kg}^{-1}$ ( Boyd $e t$ al. 2006). Ressalta-se que não há legislação ambiental brasileira vigente sobre os limites de nitrogênio e fósforo em sedimentos.

$\mathrm{O}$ crescimento em peso do tambaqui na fase berçário foi inversamente proporcional a densidade de estocagem e está de acordo com os resultados obtidos por Brandão $e t$ al. (2004). O incremento da produçáo $\left(\mathrm{kg} \mathrm{m}^{-3}\right)$ de juvenis com aumento da densidade verificado neste estudo tem sido descrito por vários autores (Gomes et al. 2006; Chagas et al. 2007; Cunha e Santos Jr. 2011). A produção média total de $14,03 \mathrm{~kg}$ foi cerca de 3,38 vezes superior a de 4,15 kg obtida na densidade de 300 peixes $\mathrm{m}^{-3}$ por Gomes et al. (2004) em tanques-rede de igual volume de $1 \mathrm{~m}^{3}$, porém com um período de criação de 60 dias inferior ao de 98 dias do presente estudo. Estes dados merecem atenção uma vez que o aumento de 38 dias no período de criação proporcionou juvenis de peso médio final de 46,8 g que superam em dobro o peso de 20,9 $\mathrm{g}$ obtido no estudo de Gomes et al. (2004). Estas diferenças podem ser atribuídas ao regime alimentar (taxa e frequência) no qual a quantidade e a qualidade do alimento são fatores determinantes nas taxas de crescimento de uma espécie em um determinado ambiente.

A baixa CAA próxima à unidade obtida na fase de recria indica que os juvenis podem utilizar com eficiência a ração oferecida desde que esta possua o balanço nutricional adequado para a espécie. Os valores da CAA (Tabela 1) são inferiores aos reportados por Silva et al. (2007) que variaram de 1,10 a 1,94 na densidade de 80 alevinos $\mathrm{m}^{-3}$ em diferentes regimes alimentares. O manejo alimentar é peça fundamental para se obter melhores CAA como o controle das sobras de ração após a oferta aos peixes para evitar a sobrealimentação e o desperdício, mas também para prevenir a deteriorização da qualidade da água pelo excesso de alimento (Mihelakakis et al. 2002). Essa estratégia de manejo foi realizada diariamente e contribuiu para os baixos valores da CAA na fase juvenil.

O peso médio final dos tambaquis obtidos na terminação foram duas vezes superiores ao de $427 \mathrm{~g}$ relatado por Chellapa et al. (1995) na densidade de 34 peixes $\mathrm{m}^{-3} \mathrm{em} 240$ dias de criação em tanques-rede na regiáo nordeste. $\mathrm{O}$ ganho médio de peso nas densidades de 20 e 40 peixes $\mathrm{m}^{-3}$ obtido nesse 
estudo (Tabela 2) foi semelhante ao publicado por Gomes et al. (2006) de 997,1 e 868,4 g, respectivamente nas mesmas densidades e período experimental inferior de 30 dias. No presente estudo aos 150 dias de criaçấo, os peixes na densidade de 20 juvenis $\mathrm{m}^{-3}$ apresentaram ganho médio de peso $(537,5$ g) superior aos relatados por Chagas et al. (2007) na densidade 15 juvenis $\mathrm{m}^{-3}$ de 320,5 e 490,4 g com taxas de alimentaçấo de 1 e $3 \%$ do peso vivo, respectivamente na região norte. A taxa de alimentação não permaneceu fixa durante a terminação como no estudo de Chagas et al. (2007) e variou entre 3\% no início e 1,2\% no final da criação. Esse maior ganho em peso pode ser atribuído ao maior teor de proteína da ração dos peixes de $32 \%$ utilizado neste estudo em relaçáo ao de $28 \%$ relatado pelos mesmos autores. O crescimento em peso é uma importante variável que deve ser monitorada e considerada na determinação da taxa de alimentação ótima. Entretanto, a eficiência na conversão do alimento também é um bom indicador, sendo que a conversão alimentar tem consequência direta nos custos de produção, pois a ração representa cerca de 30 a $60 \%$ dos custos operacionais da criação de peixes em tanque-rede (Huguenin 1997; Gomes et al. 2006).

A conversão alimentar aparente (CAA) na terminação não apresentou diferenças significativas entre os tratamentos (Tabela 2, p >0,05), diferentemente dos resultados obtidos por Gomes et al. (2006) onde as maiores densidades de 40 e 50 peixes $\mathrm{m}^{-3}$ foram mais eficientes no aproveitamento da ração. A CAA média variou de 2,11 a 2,41 (Tabela 2) e esses valores encontram-se de acordo com os obtidos por outros autores (Chellapa et al. 1995; Gomes et al. 2006; Chagas et al. 2007) em densidades similares. A taxa de crescimento específico foi inversamente proporcional à densidade e atingiu o maior valor de 0,98 na densidade de 20 juvenis $\mathrm{m}^{-3}$ (Tabela 2). Ituassú et al. (2004) publicaram valores similares da TCE de 0,90 $\pm 0,11$ para os juvenis de tambaquis que náo sofreram restrição alimentar e de 0,83 a 0,97 para os submetidos à privação alimentar, sem apresentar diferenças significativas. Por outro lado, Gomes et al. (2006) registraram valores do TCE próximos a $3 \%$ dia $^{-1}$ no segundo mês de criaçẫo provavelmente atribuído ao crescimento compensatório dos peixes e não verificaram diferenças significativas para a TCE entre as densidades de estocagem. Aride et al. (2007) observaram valores menores do TCE de 0,42 e 0,24 para o tambaqui exposto aos $\mathrm{pH}$ de 4,0 e 6,0, respectivamente. Essas diferenças na TCE são difíceis de avaliar devido às diferenças existentes entre os trabalhos de pesquisa como a fase de desenvolvimento e estado fisiológico dos peixes, condiçóes de estocagem e duração do experimento, qualidade nutricional do alimento e ração oferecida, manejo alimentar e qualidade do ambiente aquático. $\mathrm{O}$ fator de condição não apresentou diferença entre as densidades de estocagem avaliadas (Tabela 2), comportamento semelhante foi observado por Chagas et al. (2007) onde não foi encontrado diferença em tambaquis alimentados com frequências de alimentaçấo diferentes e os valores encontrados foram semelhantes $(3,4$ a 3,8$)$.

A produção foi diretamente proporcional à densidade e atingiu o valor mais alto de $48,7 \mathrm{~kg} \mathrm{~m}^{-3}$ na densidade de 60 juvenis $\mathrm{m}^{-3}$ (Tabela 2). Este é o maior patamar de produção por $\mathrm{m}^{3}$ de tambaquis em tanques-rede de acordo com os trabalhos compilados por Gomes et al. (2010). Gomes et al. (2006) obtiveram resultados similares de $45,8 \mathrm{~kg} \mathrm{~m}^{-3}$ na densidade de 60 juvenis $\mathrm{m}^{-3}$ em lagos de inundaçáo na Amazônia central. Estudos econômicos adicionais deverão ser realizados devido a importância de determinar a melhor relação custo/benefício entre a maior produção de biomassa em maiores densidades em contraposição ao maior ganho em peso em menores densidades de estocagem.

\section{CONCLUSÕES}

A densidade de estocagem recomendada para a produçáo de juvenis de tambaquis em tanques-rede é de 300 alevinos $\mathrm{m}^{-3}$. A melhor densidade para a produçáo de tambaquis de cerca de $1,0 \mathrm{~kg}$ criados em tanques-rede é de 20 juvenis $\mathrm{m}^{-3}$. A produçáo de tambaquis em tanques-rede náo alterou os níveis de nitrogênio e fósforo da água do lago abastecido com água de chuva empregando o manejo descrito neste estudo. Aumento dos teores de matéria orgânica, nitrogênio e fósforo foram verificados no sedimento abaixo dos tanques-rede, sem causar eventos de eutrofização. A criação de tambaquis em tanques-rede é viável em lagos abastecidos com água de chuva e pode integrar-se aos preceitos dos usos múltiplos da água.

\section{AGRADECIMENTOS}

A Associação dos Empregados da Embrapa pelo apoio financeiro na compra da ração utilizada no experimento, e a Companhia de Desenvolvimento dos Vales do São Francisco e do Parnaíba pela doação dos alevinos.

\section{BIBLIOGRAFIA CITADA}

Américo, J.H.P.; Torres, N.H.; Machado, A.A.M.; Carvalho, S.L.C. 2013. Piscicultura em tanques-rede: impactos e consequências na qualidade da água. Revista Cientifica ANAP Brasil, 6: 137-150.

Aguado-Giménez, F.; García-Garcia, B. 2004. Assessment of some chemical parameters in marine sediments exposed to offshore cage fish farming influence: a pilot study. Aquaculture, 242: 283-296.

APHA (American Public Health Association). 2005. Standard methods for the examination of water and waste water. APHA, Washington, DC, USA, p.4-81.

Aride, P.H.R.; Roubach, R.; Val, A.L. 2007. Tolerance response of tambaqui Colossoma macropomum (Cuvier) to water $\mathrm{pH}$. Aquaculture Research, 38: 588-594.

Boyd, C.E.; Corpron, K.; Bernard, E.; Pengsang, P. 2006. Estimates of bottom soil and effluent load of phosphorus at a semi-intensive 
marine shrimp farm. Journal of the World Aquaculture Society, 37: 41-47.

Boyd, C.E.; Tucker, C.; McNevin, A.; Bostick, K.; Clay, J. 2007. Indicators of resource use efficiency and environmental performance in fish and crustacean aquaculture. Reviews in Fisheries Science, 15: 327-360.

Brandão, F.R.; Gomes, L.C.; Chagas, E.C.; Araújo, L.D. 2004. Densidade de estocagem de juvenis de tambaqui durante a recria em tanques-rede. Pesquisa Agropecuária Brasileira, 39: 357-362.

Conte, L.; Sonoda, D.Y.; Shirota, R.; Cyrino, J.E.P. 2008. Productivity and economics of Nile tilapia Oreochromis niloticus cage culture in south-east Brazil. Journal of Applied Aquaculture, 20: $18-37$.

Costa, J.J.; Souza, R.M. 2010. Distribuição das chuvas e dinâmica geomorfológica em Barra dos Coqueiros/SE. CLIMEP Climatologia e Estudos da Paisagem, 5: 120-139.

Chagas, E.C.; Gomes, L.C.; Martins-Júnior, H.; Roubach, R. 2007. Produtividade de tambaqui criado em tanques-rede com diferentes taxas de alimentação. Ciência Rural, 37: 1109-1115.

Cunha, V.V.; Santos Junior, A. 2011. Crescimento de juvenis de tambaqui, Colossoma macropomum (CUVIER, 1818), em tanques-rede com diferentes densidades populacionais em JiParaná, RO. Amazônia: Ciência \& Desenvolvimento, 6: 185- 193.

Ferreira, D.F. 2011. Sisvar: a computer statistical analysis system. Ciência e Agrotecnologia, 35: 1039-1042.

Gomes, L.C.; Brandão, F.R.; Chagas, E.C.; Ferreira, M.F.B.; Lourenço, J.N.P. 2004. Efeito do volume do tanque-rede na produtividade de tambaqui (Colossoma macropomum) durante a recria. Acta Amazonica, 34: 111-113.

Gomes, L.C.; Chagas, E.D.; Martins-Junior, H.; Roubach, R.; Ono, E.A.; Lourenço, J.N.P. 2006. Cage culture of tambaqui (Colossoma macropomum) in a central Amazon flodplain lake. Aquaculture, 253: 374-384.

Gomes, L.C.; Simões, L.N.; Araujo-Lima, C.A.R.M. 2010. Tambaqui (Colossoma macropomum). In: Baldisserotto, B.; Gomes, L.C. (Ed.). Espécies nativas para piscicultura no Brasil. Ed. UFSM, Santa Maria, Rio Grande do Sul, p.175-204.

Henry-Silva, G.G; Camargo, A.F.M. 2006. Efficiency of aquatic macrophytes to treat nile tilapia pond effluents. Scientia Agricola, 63: 433-438.

Huguenin, J. 1997. The design, operations and economics of cage culture systems. Aquacultural Engineering, 16: 167-203.

Islam, M.S. 2005. Nitrogen and phosphorus budget in coastal and marine cage aquaculture and impacts of effluent loading on ecosystem: review and analysis towards model development. Marine Pollution Bulletin, 50: 48-61.

Ituassú, D.R.; Santos, G.R.S.; Roubach, R.; Pereira-Filho, M. 2004. Desenvolvimento de tambaqui submetido a períodos de privação alimentar. Pesquisa Agropecuária Brasileira, 39: 1199-1203.

Karakassis, I.; Tsapakis, M.; Hatziyanni, E.; Papadopoulou, K.N.; Plaiti, W. 2000. Impact of cage farming of fish on the seabed in three Mediterranean coastal areas. ICES Journal of Marine Science, 57: 1462-1471.
La Rosa, T.; Mirto, S.; Mazzola, A.; Maugeri, T.L. 2004. Benthic microbial indicators of fish farm impact in a coastal area of the Thyrrenian Sea. Aquaculture, 230: 153-167.

Mallasen, M.; Carmo, C.F.; Tucci, A.; Barros, H.P.; Rojas, N.E.T.; Fonseca, F.S.; Yamashita, E.Y. 2012. Qualidade da água em sistema de piscicultura em tanques-rede no reservatório de Ilha Solteira, SP. Boletim do Instituto de Pesca, 38: 15-30.

Mihelakakis, A.; Tsolkas, C.; Yoshimatsu, T. 2002. Optimization of feedind rate of hatchery produced juvenile gilthead sea bream Sparus aurata. Journal of the World Aquaculture Society, 33: 169-175.

Molina, L.; López, G.; Vergara, J.M.; Robaina, L. 2001. A comparative study of sediments under a marine cage farm at Canary Island (Spain). Preliminary results. Aquaculture, 192: 225-231.

Pillay, T.V.R. 2004. Waste production in aquaculture. In: Pillay, T.V.R. (Ed.). Aquaculture and the environment. Blackwell Publishing, Noida, p.58-75

Primavesi, A.C.; Andrade, A.G.; Alves, B.J.R.; Rosso, C.; Batista, E.M.; Prates, H.T. 2005a. Água. In: Nogueira, A.R.A.; Souza, G.B. (Ed.). Manual de Laboratórios: solo, água, nutrição vegetal, nutrição animal e alimentos. Embrapa Pecuária Sudeste, Sáo Carlos, São Paulo, p.131-144.

Primavesi, A.C.; Andrade, A.G.; Alves, B.J.R.; Rosso, C.; Batista, E.M.; Prates, H.T. 2005b. Métodos de análise de solo. In: Nogueira, A.R.A.; Souza, G.B. (Ed.). Manual de Laboratórios: solo, água, nutrição vegetal, nutrição animal e alimentos. Embrapa Pecuária Sudeste, São Carlos, São Paulo, p.67-130.

SIRHSE, 2012. Sistema de Informação sobre Recursos Hídricos (http://sirhse.semarh.se.gov.br/sirhse/index.php/ macroplanejamento/bacias_hidrograficas/estadoClima/estado). Acesso em 06/03/2013.

Silva, C.A. 2011. Boas Práticas de Manejo na criação de tambaqui em viveiro no Baixo São Francisco. Circular técnica Embrapa, 64: 1-8.

Silva, C.R.; Gomes, L.C.; Brandão, F.R. 2007. Effect of feeding rate and frequency on tambaqui (Colossoma macropomum) growth, production and feeding costs during the first growth phase in cages. Aquaculture, 264: 135-139.

Silva, A.D.R.; Santos, R.B.; Bruno, A.M.S.S.; Soares, E.C. 2013. Cultivo de tambaqui em canais de abastecimento sob diferentes densidades de peixes. Acta Amazonica, 43: 517-524.

Silva, A.E.P.; Angelis, C.F.; Machado, L.A.T.; Waichaman, A.V. 2008. Influência da precipitaçáo na qualidade da água do Rio Purus. Acta Amazonica, 38: 733-742.

Rowland, S. J.; Mifsuda, C.; Nixon, M.; Boyd, P. 2006. Effects of stocking density on the performance of the Australian freshwater silver perch (Bidyanus bidyanus) in cages. Aquaculture, 253: 301-308.

Recebido em 29/05/2014

Aceito em 20/01/2015 
